\title{
A Note on the Asymptotic and Threshold Behaviour of Discrete Eigenvalues inside the Spectral Gaps of the Difference Operator with a Periodic Potential
}

\author{
Gift Muchatibaya $(\mathbb{D}$ and Josiah Mushanyu \\ Department of Mathematics, University of Zimbabwe, MP 167, Mt Pleasant, Harare, Zimbabwe \\ Correspondence should be addressed to Gift Muchatibaya; giftmuchatibaya@gmail.com
}

Received 1 August 2017; Revised 10 January 2018; Accepted 28 January 2018; Published 22 February 2018

Academic Editor: Antonio Scarfone

Copyright (c) 2018 Gift Muchatibaya and Josiah Mushanyu. This is an open access article distributed under the Creative Commons Attribution License, which permits unrestricted use, distribution, and reproduction in any medium, provided the original work is properly cited.

The asymptotic and threshold behaviour of the eigenvalues of a perturbed difference operator inside a spectral gap is investigated. In particular, applications of the Titchmarsh-Weyl $m$-function theory as well as the Birman-Schwinger principle is performed to investigate the existence and behaviour of the eigenvalues of the operator $H_{0}+\lambda W_{n}$ inside the spectral gap of $H_{0}$ in the limits $\lambda \uparrow \infty$ and $\lambda \downarrow 0$.

\section{Introduction}

We consider the Schrödinger difference equation

$$
H_{\lambda} u=\left(H_{0}+\lambda W_{n}\right) u_{n}=E u_{n}, \quad n \in[0, \infty),
$$

in the Hilbert space $l^{2}(\mathbb{Z})$ of all square summable sequences $\left\{x_{n}\right\}_{1}^{\infty}$ such that $\sum_{1}^{\infty}\left|x_{n}\right|^{2}<\infty$. Here, $H_{0}=-\Delta+$ $V_{n}$, the Laplacian $-\Delta$ is a second-order finite difference operator given by $(-\Delta u)(n):=-u_{n+1}+2 u_{n}-u_{n-1}$. The operator $H_{0}$ describes a well-understood system with $V_{n}$ being the background periodic or quasi-periodic potential. The parameter $\lambda$, called the coupling constant, is real and positive and $E$ is a complex parameter. The sequences $\left\{V_{n}\right\}_{0}^{\infty}$ are real and are such that $V_{a+n}=V_{a}$, for some $a \in \mathbb{Z}^{+}$. The perturbing term $W_{n}$ satisfies the scattering condition

$$
\sum_{n=0}^{\infty} n\left|W_{n}\right|<0 .
$$

The domain $D_{H_{\lambda}}$ of $H_{\lambda}$ consists of all $x \in l^{2}(\mathbb{Z})$ such that $H_{\lambda} x \in l^{2}(\mathbb{Z})$. The operators $H_{\lambda}$ and $H_{0}$ are known to be selfadjoint $[1,2]$.

Operators of the form $H_{\lambda}=H_{0}+\lambda W$, either in the difference or in the continuum form, occur frequently in quantum mechanics as mathematical models of Schrödinger type that models semiconductors with impurities $[1,3]$. The impurity levels in solids reduce the width of the gap where they are situated, and this feature has important consequences from the point of view of conductivity properties of the solids. Furthermore, such impurity levels lead to a selective absorption of certain photon energies which is an important element in the theory of crystal colours. Perhaps the most appealing example in nature is the $\mathrm{Al}_{2} \mathrm{O}_{3}$ crystal, which has a large gap between the first and the second bands: it is transparent and colourless. By replacing the $\mathrm{Al}^{+}$-ion by $\mathrm{Cr}^{3+}$, one obtains a familiar complementary colour red, and this is due to the fact that the impurity levels lead to the absorption of green light $[4,5]$. Recent and relevant results on the application of the solutions of Schrödinger equations with physical potential models in the field of thermal physics, for example, can be found in the work of Jia et al. [6-9].

The spectrum $\rho\left(H_{0}\right)$ of the self-adjoint operator $H_{0}$ is real and consists of two absolutely continuous bands separated by a gap. By a spectral gap of $H_{0}$, we mean an interval $(a, b)$ such that $a<b, a, b \in \rho\left(H_{0}\right)$, and $(a, b) \cap \rho\left(H_{0}\right) \neq \emptyset$. We choose our $V_{n}$ 's such that the gaps are empty when $\lambda=0$. For $\lambda>0$, the spectrum of $H_{\lambda}$ is made up of the continuous part which coincides with that of $\rho\left(H_{0}\right)$ together with at most a finite number of eigenvalues inside the gap of $\rho\left(H_{0}\right)$ 
$[3,10-12]$. Of interest to this work are the conditions on $W_{n}$ guaranteeing the existence of such eigenvalues and their asymptotic behaviour as $\lambda$ varies.

The case where $V_{n}$ is not periodic but decays fast enough to zero as $n \rightarrow 0$ was studied in [13], and a formula of the type known as Levinson's theorem was derived which counts the number of eigenvalues or bound states outside the continuous band $[0,4]$. We would like to obtain the corresponding results when $V_{n}$ is periodic and determine how those eigenvalues vary as the coupling constant becomes large and small. Some results do exist for the Schrödinger case; however, in the discrete case this has not been looked at (see, e.g., [3]).

Since there are no eigenvalues when $\lambda=0$, by general results of perturbation theory, eigenvalues of $H_{\lambda}$ can appear in a gap only by emerging from one of its end points as $\lambda$ is varied. Likewise, eigenvalues can disappear from the gap only by converging to an end point [14-18]. We call $\lambda_{0}$ a coupling constant threshold of the family of the operators $H_{\lambda}$ at the gap end points $a$ and $b$ if there exists an eigenvalue branch $E(\lambda)$ of $H_{\lambda}$ such that $E(\lambda) \downarrow a$ or $E(\lambda) \uparrow b$, as either $\lambda \uparrow \lambda_{0}$ or $\lambda \downarrow \lambda_{0}$, respectively, or both. In particular, the case where $E(\lambda) \downarrow a$ as $\lambda \downarrow \lambda_{0}$ corresponds to the situation where an eigenvalue appears at $a$ as $\lambda \downarrow \lambda_{0}+\epsilon$. For more results in connection with the Schrödinger case, see, for example, [3].

The purpose of this work is to (1) study the analytic behaviour of the eigenvalue $E(\lambda)$ near the coupling constant threshold and (2) study the asymptotic behaviour of the discrete spectrum in the gaps of the spectrum of $H_{0}$ as the coupling constant grows to infinity. Our main tool will be the Birman-Schwinger operator and the Titchmarsh-Weyl $m$ function theory.

\section{The Birman-Schwinger Principle}

The spectrum $\rho\left(H_{0}\right)$ of $H_{0}$ is real and consists of two absolutely continuous bands covering the closed intervals $\left[E_{0}, E_{1}\right] \cup\left[E_{2}, E_{3}\right]$, where $0<E_{0}<E_{1}<E_{2}<E_{3}$. Since $W_{n}$ is assumed to be relatively $H_{0}$-compact, we also have that $\rho_{\text {ess }}\left(H_{0}+\lambda W_{n}\right)=\rho_{\text {ess }}\left(H_{0}\right)$.

If we let $E(\lambda) \in\left(E_{1}, E_{2}\right)$, then the Birman-Schwinger kernel is defined by $[19,20]$

$$
K_{E}=\left|W_{n}\right|^{1 / 2}\left(H_{0}-E\right) W_{n}^{1 / 2}
$$

The Birman-Schwinger principle implies the following.

Let $H_{0}$ be a self-adjoint operator and $E \in \rho\left(H_{0}\right)$, and suppose that $W_{n} \geq 0$ is a bounded operator with $W_{n}\left(H_{0}-E\right)^{-1}$ compact. Then the Birman-Schwinger kernel $K_{E}$ is compact and the following are equivalent:

(1) $E$ is an eigenvalue of $H_{\lambda}$ with multiplicity $m$.

(2) $\lambda^{-1}$ is an eigenvalue of $K_{E}$ with multiplicity $m$.

The following results are well known, we state them without proof, and the proofs for the continuous case can be found in [3], for example.

Proposition 1. Suppose that $W_{n} \leq 0\left(W_{n} \geq 0\right)$, and then the nonzero eigenvalues of $K_{E}$ are strictly monotone increasing (or decreasing) respectively.
Proposition 2. There exists a $\lambda \in \mathbb{R}$ such that $H_{0}+\lambda W_{n}$ has an eigenvalue in $\left(E_{1}, E_{2}\right)$.

The following lemma summarizes all the information concerning the operator $K_{E}$ and the behaviour of the eigenvalues inside the spectral gap, and its proof can be found in [3].

Lemma 3. Let $W_{n} \leq 0\left(W_{n} \neq 0\right)$. Then

(1) if $\lambda=0$ is a coupling constant threshold of $H_{0}+\lambda W_{n}$ then $\left\|K_{E}\right\|$ is unbounded as $E \uparrow E_{2}$,

(2) if $\left\|K_{E}\right\|$ stays bounded as $E \uparrow E_{2}$, then $\lim _{E \uparrow E_{2}} K_{E}=$ $K_{E_{2}}$ exists,

(3) $K_{E_{2}}$ is compact iff $K_{E} \rightarrow K_{E_{2}}$ in norm,

(4) if $\operatorname{dim} E_{\left(E_{1}, E_{2}\right)}\left(H_{0}+\lambda W_{n}\right)=\infty$ for some $\lambda=\lambda_{1}>0$ then $\operatorname{dim} E_{\left(E_{1}, E_{2}\right)}\left(H_{0}+\lambda W_{n}\right)=\infty$ for all $\lambda>\lambda_{1}$,

(5) $K_{E_{2}}$ is compact iff $\operatorname{dim} E_{\left(E_{1}, E_{2}\right)}\left(H_{0}+\lambda W_{n}\right)<\infty$ for all $\lambda>0$,

(6) suppose that $\lambda=0$ is not a coupling constant threshold and $\operatorname{dim} E_{\left(E_{1}, E_{2}\right)}\left(H_{0}+\lambda W_{n}\right)<\infty$ for all $\lambda>0$. Then $K_{E_{2}}$ is compact,

(7) $K_{E_{2}}$ exists and is not compact iff $\lambda=0$ is not a coupling constant threshold and there exists $\lambda^{*}>0$ such that $\operatorname{dim} E_{\left(E_{1}, E_{2}\right)}\left(H_{0}+\lambda W_{n}\right)=\infty$ for $\lambda>\lambda^{*}$ and $\operatorname{dim} E_{\left(E_{1}, E_{2}\right)}\left(H_{0}+\lambda W_{n}\right)<\infty$ for $\lambda<\lambda^{*}$.

The threshold behaviour is described in the following lemma.

Lemma 4. Suppose that $W_{n} \leq 0\left(W_{n} \neq 0\right)$ and suppose $\lambda=0$ is not a threshold. Then $E_{2}$ is not an eigenvalue of $H_{0}$ iff all eigenvalues $E_{i}(\lambda)$ which are absorbed at $\lambda=0$ obey $\left(E_{i}(\lambda)-\right.$ $\left.E_{2}\right) \lambda^{-1} \rightarrow 0$.

\section{The $m(E)$-Function Theory}

Let $\theta_{n}(E, \lambda)$ and $\phi_{n}(E, \lambda)$ be the two linearly independent solutions of (1) satisfying the following initial conditions for all $E \in \mathbb{C}$ :

$$
\begin{aligned}
& \theta_{0}(E, \lambda)=\phi_{1}(E, \lambda)=1, \\
& \theta_{1}(E, \lambda)=\phi_{0}(E, \lambda)=1,
\end{aligned}
$$

and then there exists a solution $\psi_{n}(E, \lambda)=\theta_{n}(E, \lambda)+$ $m(E, \lambda) \phi_{n}(E, \lambda)$, which is in $l^{2}(0, \infty)$. The function $m(E, \lambda)$ is defined by

$$
m(E, \lambda)=-\lim _{n \rightarrow \infty} \frac{\theta_{n}(E, \lambda)}{\phi_{n}(E, \lambda)} .
$$

The general proof for the existence of the limit in (5) may be found in $[1,21]$. The $m(E)$-function is analytic for $\operatorname{Im}(E) \neq$ 0 , and it has a nonreal $\operatorname{limit}$ as $\operatorname{Im}(E) \rightarrow 0$ in the bands and is real in the gaps except for the poles of $m(E)$ at the eigenvalues of $H_{\lambda}$. Of interest is the behaviour of $m(E)$ at the end points of the gaps, if the endpoints are either halfbound states or otherwise. $E(\lambda)=E_{1}$ is a half-bound state 
(HBS) provided that $\left\{\phi_{n}(E)\right\}$ is abounded sequence but not in $l^{2}(0, \infty)$ and a non-half-bound state (non-HBS) otherwise. A similar definition may be given at the other end point of the spectral gap. In [13], there are the results of a version of Levinson's Theorem for the system

$$
-u_{n+1}+2 u_{n}-u_{n-1}+V_{n} u_{n}=E u_{n}, \quad n \in[0, \infty),
$$

with $\sum_{0}^{\infty} n\left|V_{n}\right|<\infty$.

Imposing the boundary conditions $u_{0}+\alpha u_{1}=0$, the spectrum of $(6)$ is $[0,4]$, with a finite number of eigenvalues in the intervals $(-\infty, 0) \cup(4, \infty)$. At an eigenvalue $E_{0} \in$ $(-\infty, 0) \cup(4, \infty)$, we have that

$$
\left(E-E_{0}\right) m(E) \longrightarrow k_{1} \neq 0 \text { as } E \longrightarrow E_{0} .
$$

At the points $E=0,4$ it turns out that

$$
\begin{aligned}
& \left(E-E_{i}\right)^{1 / 2} m(E) \\
& \longrightarrow \begin{cases}k_{2} \neq 0, & \text { if } E_{i}=0 \text { or } 4 \text { is HBS } \\
0, & \text { else. }\end{cases}
\end{aligned}
$$

It is natural to ask whether the theory of [13] carries over to the periodic version (1) in the presents of a gap and possible half-bound states at the ends of each gap.

\section{The Bound States of $H_{0}+\lambda W_{n}$}

We consider the following system:

$$
\begin{aligned}
\left(H_{0}+\lambda W_{n}\right) u_{n} & =-u_{n+1}+2 u_{n}-u_{n-1}+\left(V_{n}+\lambda W_{n}\right) u_{n} \\
& =E u_{n}, \quad u_{n} \in l^{2}(\mathbb{Z}),
\end{aligned}
$$

where $V_{n+a}=V_{n}$, for some $a \in \mathbb{Z}$, and $\sum|n| W_{n}<\infty$. Let $\theta_{n}, \phi_{n}$ be the solutions of $H_{0} u_{n}=E u_{n}$ satisfying the following initial conditions: $\theta_{0}=1, \theta_{1}=0$, and $\phi_{0}=0, \phi_{1}=1$

Let

$$
\psi(n)=\left(\begin{array}{cc}
\theta_{n} & \phi_{n} \\
\theta_{n+1} & \phi_{n+1}
\end{array}\right), \quad \text { i.e } \psi(0)=\left(\begin{array}{ll}
1 & 0 \\
0 & 1
\end{array}\right),
$$

and this defines the fundamental matrix for $\left(H_{0}-E\right) u=0$, and moreover $\psi(n+a)=\psi(n) \psi(a)$ and $\operatorname{det} \psi(a)=\operatorname{det} \psi(0)=$ 1. The eigenvalues of $\psi(a)$ are

$$
\mu_{ \pm}=\frac{D \pm \sqrt{D^{2}-4}}{2}, \quad D=\theta_{a}+\phi_{a+1} .
$$

The gaps and bounds are given by $|D(E)|>2$ and $|D(E)|>2$, respectively. Let us just look at the specific situation where $E$ is near $E_{1}$. We set

$$
\alpha=\ln \mu_{+}=\ln \frac{D+\sqrt{D^{2}-4}}{2},
$$

and notice that we can view $E$ as an even function of $\alpha$ in the vicinity of $E_{1}$; that is, for $\alpha$ small

$$
\begin{aligned}
\sqrt{D^{2}-4} & =\sqrt{\left[2+c\left(E-E_{1}\right)+\cdots\right]^{2}-4} \\
& =\sqrt{2|c|} \sqrt{E_{1}-E\left(1+\text { powers of }\left(E-E_{1}\right)\right)},
\end{aligned}
$$

so that $E$ goes around $E_{1}, \sqrt{D^{2}-4} \rightarrow-\sqrt{D^{2}-4}$, and $\mu_{+} \rightarrow-\mu_{-}$, that is, $\alpha \rightarrow-\alpha$. Denoting the eigenfunctions corresponding to $\mu_{ \pm}$by $q_{ \pm}$where

$$
q_{ \pm}=\left(1, \frac{\mu_{ \pm}-\theta_{a}}{\phi_{a}}\right),
$$

provided $\phi_{a} \neq 0$ (if $\phi_{a}=0$, we shift the origin), we now define the periodic vector functions

$$
\widetilde{q_{ \pm}}(n)=\psi(n) q_{ \pm}, \quad 0 \leq n \leq a-1,
$$

with $\widetilde{q_{ \pm}}(n)=\widetilde{q_{ \pm}}(n+a)$. Let

$$
\begin{aligned}
f_{n} & = \begin{cases}0, & 0 \leq n \leq a-1 \\
f_{n+a}=f_{n}+1, & \text { elsewhere, }\end{cases} \\
\widetilde{p_{ \pm}}(n) & =e^{( \pm f(n) \mp n / a) \alpha} \widetilde{q_{ \pm}}(n),
\end{aligned}
$$

and it follows that $\widetilde{p_{ \pm}}(n+a)=\widetilde{p_{ \pm}}(n)$. Let

$$
\Psi^{ \pm}=\phi(n) q_{ \pm}(n)=e^{ \pm(n / a) \alpha} \widetilde{p_{ \pm}}(n) .
$$

These are the exponential solutions which behave in the following way:

$$
\Psi^{ \pm} \longrightarrow 0 \text { as } n \longrightarrow \mp \infty
$$

As for the Wronskian $\widetilde{W}=W\left(\Psi^{+}, \Psi^{-}\right)$, we have

$$
\widetilde{W}=\frac{\mu_{-}-\mu_{+}}{\phi_{a}}=-\frac{\sqrt{D^{2}-4}}{\phi_{a}} .
$$

We have that $\phi_{a}<0$ (at the right endpoint of a gap where $D(E)>2$ ). This follows from general principles below since when $W_{n}<0$, the eigenvalues in the gap must appear at the right endpoint if $E$ increases. It follows directly in this way: if we write $\Psi^{-}=\theta+m \phi$, where $m=\left(\mu_{-}-\theta_{a}\right) / \phi_{a}$, then $m(E)$ is the Titchmarsh-Weyl function. In the Schrödinger case $\operatorname{Im} m(E)$ is positive for $\operatorname{Im} E>0$. Let us see what it is here: from

$$
\begin{aligned}
& u_{n+1}-2 u_{n}+u_{n-1}+V_{n} u_{n}=E u_{n}, \\
& \bar{u}_{n+1}-2 \bar{u}_{n}+\bar{u}_{n-1}+V_{n} \bar{u}_{n}=\bar{E} \bar{u}_{n},
\end{aligned}
$$

summing from 1 to $\infty$ gives

$$
u_{0} \bar{u}_{1}-\bar{u}_{0} u_{1}=(E-\bar{E}) \sum_{1}^{\infty} u_{n} \bar{u}_{n}
$$

where $u_{0} \bar{u}_{1}-\bar{u}_{0} u_{1}=\bar{m}(E)-m(E)$ so that $\operatorname{Im} m(E)<0$ when $\operatorname{Im}(E)>0$. If $E$ is close to $E_{1}$ in the upper half plane, then $\operatorname{Im} \mu_{-}>0$ and hence $\psi_{a}<0$.

Now let us consider the Birman-Schwinger kernel:

$$
K_{E}=\left|W_{n}\right|^{1 / 2}\left(H_{0}-E\right) W_{n}^{1 / 2} \text {, }
$$


and, assuming $W_{n} \leq 0, K_{E}$ has eigenvalue 1 iff $E \in \rho(H)$. The resolvent $\left(H_{0}-E\right)^{-1}$ is given by

$$
\begin{aligned}
\left(\left(H_{0}-E\right)^{-1} u\right)_{n} & =\frac{1}{\bar{W}} \Psi_{n}^{-} \sum_{-\infty}^{n-1} \Psi_{m}^{+} u_{m}+\frac{1}{\bar{W}} \Psi_{n}^{+} \sum_{n}^{\infty} \Psi_{m}^{-} u_{m} \\
& =\sum R_{n m} u_{m},
\end{aligned}
$$

with

$$
R_{m n}=\frac{1}{\bar{W}} \Psi_{n}^{-} \Psi_{m}^{+} \chi_{n \geq m+1}+\frac{1}{\bar{W}} \Psi_{n}^{+} \Psi_{m}^{-} \chi_{m \geq n}
$$

Using (17), we get (using $\alpha$ as a variable)

$$
\begin{aligned}
R_{m n}= & \frac{1}{\bar{W}} e^{-\alpha|n-m|} p_{-}(n, \alpha) p_{+}(m, \alpha) \chi_{n \geq m+1} \\
& +\frac{1}{\bar{W}} e^{-\alpha|n-m|} p_{+}(n, \alpha) p_{-}(m, \alpha) \chi_{m \geq n},
\end{aligned}
$$

where $p_{ \pm}(n, \alpha)$ is the first component of $\widetilde{p_{ \pm}}(n) \cdot p_{+}(n, \alpha)$ is analytic in $\alpha$ with $p_{+}(n, \alpha)=p_{+}(n,-\alpha)$. We note that

$$
\widetilde{W}=-\frac{2 \sinh \alpha}{\psi_{a}}>0, \quad\left(E<E_{1}\right) .
$$

We aim to determine the limit as $\alpha \rightarrow 0$ of $R_{m n}$. There is a diverging rank one piece which we can isolate.

$$
\begin{aligned}
& R_{m n} \\
& =\frac{1}{\bar{W}} e^{-\alpha|n|} p_{-}(n, 0) e^{-\alpha|m|} p_{+}(m, 0) \\
& \quad+\frac{1}{\bar{W}}\left[e^{-\alpha|n-m|}-e^{-\alpha|n|} e^{-\alpha|m|}\right] p_{+}(n, 0) p_{+}(m, 0) \\
& \quad+M_{m n}+N_{m n},
\end{aligned}
$$

where

$$
\begin{gathered}
M_{m n}=\frac{1}{\bar{W}} e^{-\alpha|n-m|}\left[p_{-}(n, \alpha) p_{+}(m, \alpha)\right. \\
\left.-p_{-}(n, 0) p_{+}(m, 0)\right] \chi_{n \geq m+1}, \\
N_{m n}=\frac{1}{\bar{W}} e^{-\alpha|n-m|}\left[p_{+}(n, \alpha) p_{-}(m, \alpha)\right. \\
\left.-p_{+}(n, 0) p_{-}(m, 0)\right] \chi_{m \geq n} .
\end{gathered}
$$

The first term gives a rank one contribution to $K_{E}$ and it diverges as $E \uparrow E_{1}$, with $\lambda \uparrow \infty$. The other terms when sandwiched between $|W|^{1 / 2}$ go to finite limits in Hilbert Schmidt norm. The rank one piece gives rise to a bound state near $E_{1}$ in the weak coupling limit (if $\sum W_{n} e^{-2 \alpha|n|} p_{+}(n, 0)^{2} \leq$ 0 , even if $W_{n}$ changes sign). If $\sum W_{n} e^{-2 \alpha|n|} p_{+}(n, 0)^{2} \geq 0$, then the eigenvalue gets absorbed at $E=E_{2}$ as $\lambda \downarrow 0$.

\section{Conclusion}

By using the standard results derived for the continuous Schrödinger operator case, some insights into the asymptotic behaviour of eigenvalues of the difference Schrödinger operator in the limit of the large and small coupling constant have been derived. This forms the basis for future work on the investigation of the asymptotic behaviour of the number of the eigenvalues inside the spectral gap for a large coupling constant which are not covered in this note.

\section{Conflicts of Interest}

The authors declare that there are no conflicts of interest regarding the publication of this paper.

\section{Acknowledgments}

The authors would like to thank Professor Martin Klaus (Virginia Tech) for several useful discussions at the time when this paper was conceived of. Gift Muchatibaya and Josiah Mushanyu would like to acknowledge the support of the University of Zimbabwe when this project was underway.

\section{References}

[1] T. Asahi, "Spectral theory of the difference equations," Progress of Theoretical and Experimental Physics, vol. 36, pp. 55-96, 1966.

[2] H. Behncke and F. O. Nyamwala, "Spectral theory of difference operators with almost constant coefficients," Journal of Difference Equations and Applications, vol. 17, no. 5, pp. 677-695, 2011.

[3] S. Fassari and M. Klaus, "Coupling constant thresholds of perturbed periodic Hamiltonians," Journal of Mathematical Physics, vol. 39, no. 9, pp. 4369-4416, 1998.

[4] P. A. Deift and R. Hempel, "On the existence of eigenvalues of the Schrödinger operator $H-\lambda W$ in a gap of $\sigma(H)$, Commun," Communications in Mathematical Physics, vol. 103, no. 3, pp. 461-490, 1986.

[5] W. M. Elliot and B. P. Renfrey, "Effects of defects on lattice vibrations," Phys Review, vol. 100, pp. 525-543, 1955.

[6] C. Jia, L. Zhang, and C. Wang, "Thermodynamic properties for the lithium dimer," Chemical Physics Letters, vol. 667, pp. 211215, 2017.

[7] C. Jia, C. W. Wanga, L. H. Zhanga, X. L. Penga, R. Zenga, and X. T. Youa, "Partition function of improved Tietz oscillators," Chemical Physics Letters, vol. 676, pp. 150-153, 2017.

[8] J.-F. Wang, X.-L. Peng, L.-H. Zhang, C.-W. Wang, and C.-S. Jia, "Entropy of gaseous boron monobromide," Chemical Physics Letters, vol. 686, pp. 131-133, 2017.

[9] X. Q. Songa, C. W. Wanga, and C. S. Jia, "Thermodynamic properties for the sodium dimer," Chemical Physics Letters, vol. 673 , pp. 50-55, 2017.

[10] M. S. Birman, "Discrete spectrum in the gaps of the continuous one in the large-coupling-constant limit," in Order, Disorder and Chaos in Quantum Systems. Operator Theory: Advances and Applications, vol. 46, pp. 17-25, Birkhauser Basel, 1990.

[11] M. S. Birman, "The discrete spectrum in the gaps of a secondorder perturbed periodic operator," Functional Analysis and its Applications, vol. 25, no. 2, pp. 158-161, 1991.

[12] R. F. Wallis, "Effect of free ends on the vibration frequencies of one-dimensional lattices," Physical Review A: Atomic, Molecular and Optical Physics, vol. 105, no. 2, pp. 540-545, 1957. 
[13] D. B. Hinton, M. Klaus, and J. K. Shaw, "Half-bound states and Levinson's theorem for discrete systems," SIAM Journal on Mathematical Analysis, vol. 22, no. 3, pp. 754-768, 1991.

[14] S. Alama, P. A. Deift, and R. Hempel, "Eigenvalue branches of the Schrödinger operator $H-\lambda W$ in a gap of $\sigma(H)$, Commun," Communications in Mathematical Physics, vol. 121, no. 2, pp. 291-321, 1989.

[15] K. Kumar, M. N. Namboodiri, and S. Serra-Capizzano, "Perturbation of operators and approximation of spectrum," The Proceedings of the Indian Academy of Sciences - Mathematical Sciences, vol. 124, no. 2, pp. 205-224, 2014.

[16] K. M. Schmidt, "Critical coupling constants and eigenvalue asymptotics of perturbed periodic Sturm-Liouville operators," Communications in Mathematical Physics, vol. 211, no. 2, pp. 465-485, 2000.

[17] A. V. Sobolev, "The asymptotic behaviour of the discrete spectrum in the gaps of the continuous spectrum of a perturbed Hill operator," Functional Analysis and Its Applications, vol. 25, no. 2, pp. 93-95, 1991.

[18] V. A. Zheludev, "Perturbation of the one-dimensional selfadjoint Schrödinger operator with a periodic potential," Topics in Mathematical Physics, vol. 2, pp. 55-75, 1968.

[19] M. Klaus, "Some applications of the Birman-Schwinger principle," Helvetica Physica Acta, vol. 55, no. 1, pp. 413-419, 1982/83.

[20] M. Klaus, "On the bound state of Schrödinger operators in one dimension," Annals of Physics, vol. 108, no. 2, pp. 288-300, 1977.

[21] W. N. Everitt and S. G. Halvorsen, "On the asymptotic form of the Titchmarsh-Weyl $m$-function coefficient," Applicable Analysis: An International Journal, vol. 8, no. 2, pp. 153-169, 1978. 


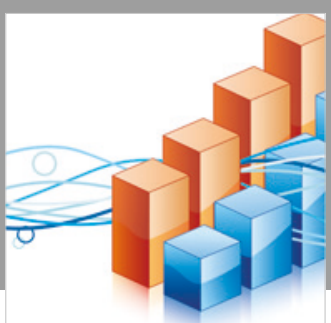

Advances in

Operations Research

\section{-n-m}
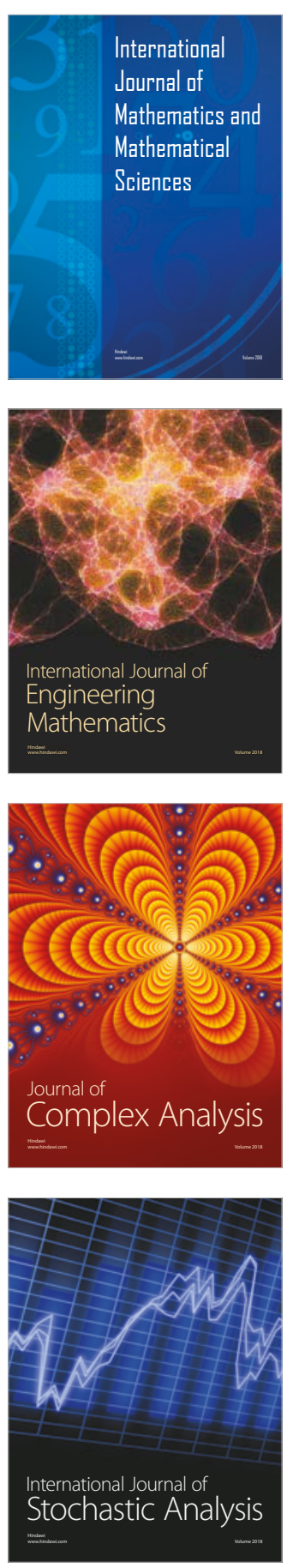
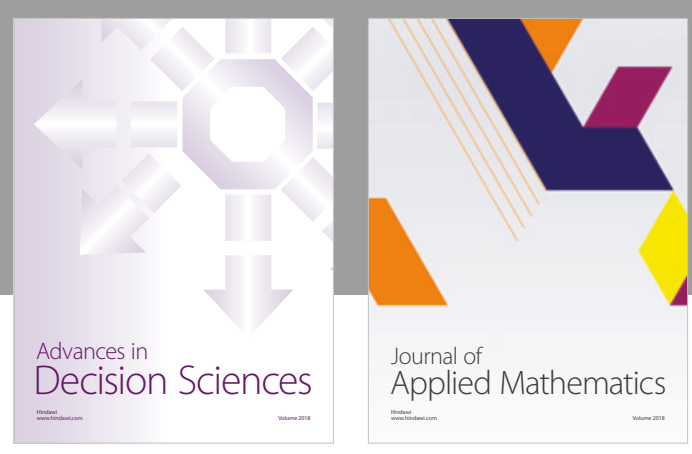

Journal of

Applied Mathematics
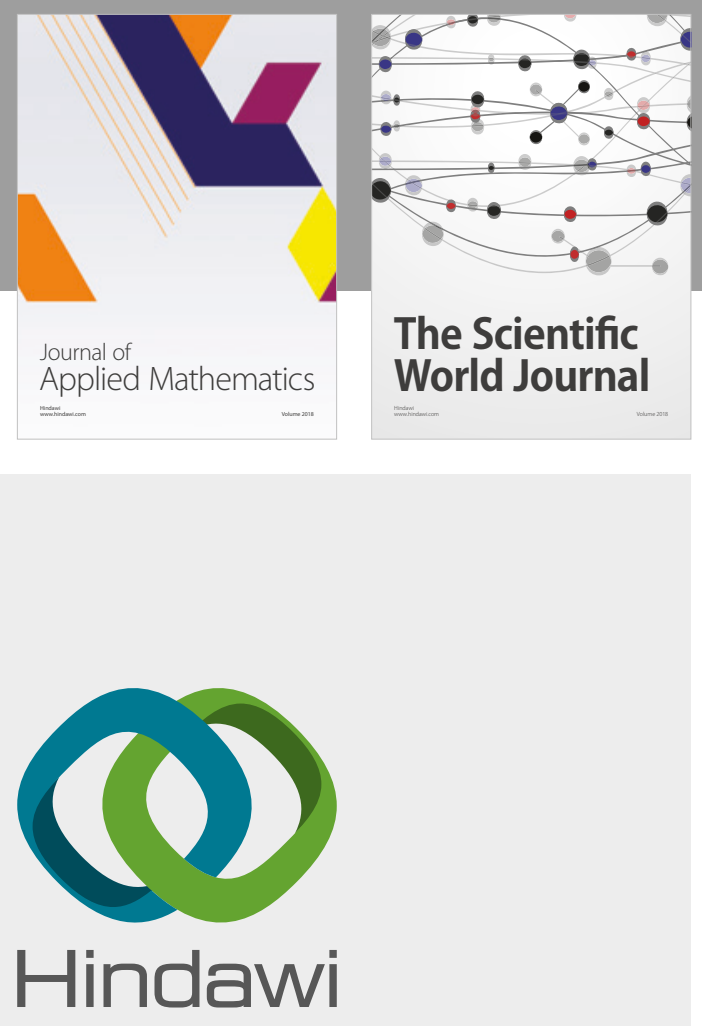

Submit your manuscripts at

www.hindawi.com

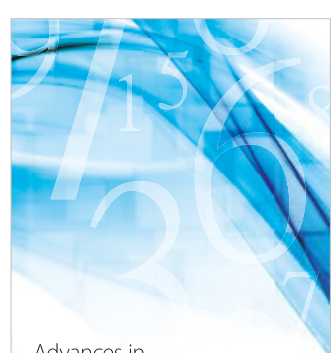

Advances in
Numerical Analysis
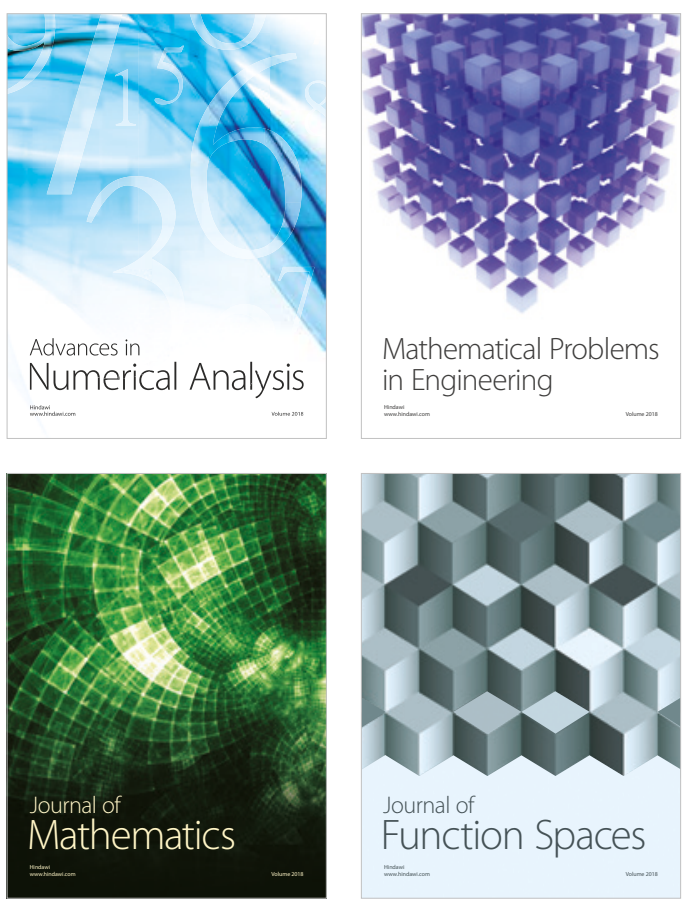

Mathematical Problems in Engineering

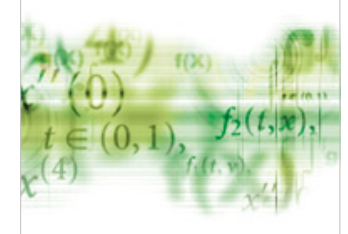

International Journal of

Differential Equations

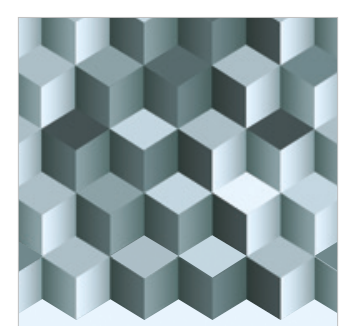

Journal of

Function Spaces

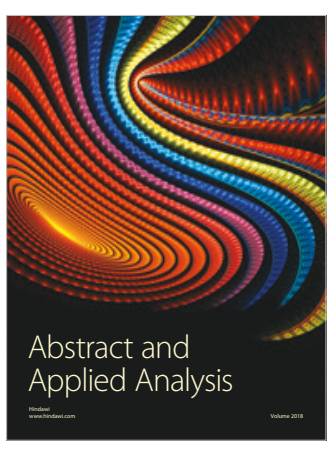

The Scientific

World Journal

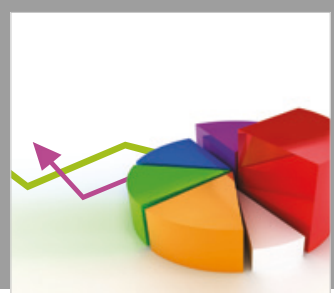

Journal of

Probability and Statistics
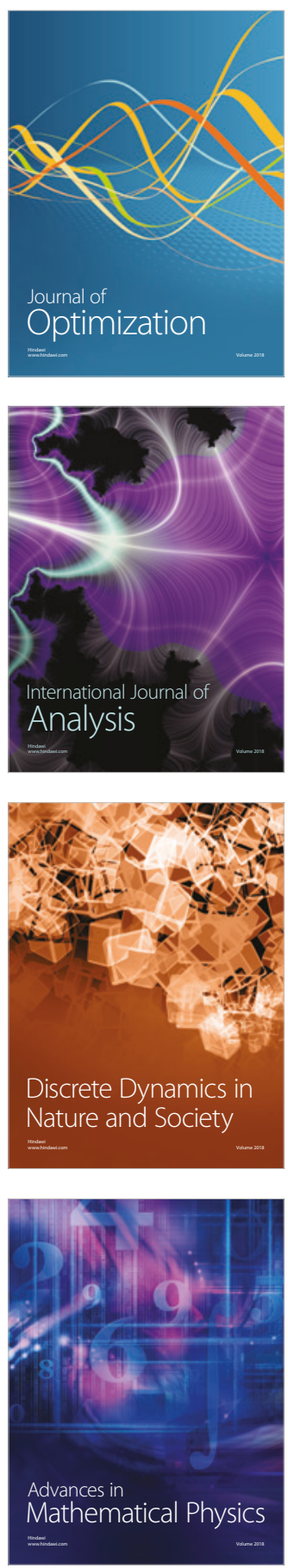